\title{
Drying Method
}

National Cancer Institute

\section{Source}

National Cancer Institute. Drying Method. NCI Thesaurus. Code C112923.

A process that removes water or volatile solvents from a bed of solids. 